EPJ Web of Conferences 64, 01004 (2014)

DOI: $10.1051 /$ epjconf/ 20146401004

(C) Owned by the authors, published by EDP Sciences, 2014

\title{
Swinging between rotation and accretion power in a binary millisecond pulsar
}

\author{
A. Papitto ${ }^{1, a}$, C. Ferrigno ${ }^{2}$, E. Bozzo ${ }^{2}$, and N. Rea ${ }^{1,3}$ \\ ${ }^{1}$ Institute of Space Sciences (ICE; IEEC-CSIC), Campus UAB, Faculty of Science, Torre C5, parell, 2a \\ planta, E-08193 Barcelona, Spain \\ ${ }^{2}$ ISDC Data Center for Astrophysics, Université de Genève, chemin d'Écogia, 16, CH-1290 Versoix, \\ Switzerland \\ ${ }^{3}$ Astronomical Institute "Anton Pannekoek", University of Amsterdam, Science Park 904, 1098 XH \\ Amsterdam, The Netherlands
}

\begin{abstract}
We present the discovery of IGR J18245-2452, the first millisecond pulsar observed to swing between a rotation-powered, radio pulsar state, and an accretion-powered $\mathrm{X}$-ray pulsar state [31]. This transitional source represents the most convincing proof of the evolutionary link shared by accreting neutron stars in low mass X-ray binaries, and radio millisecond pulsars. It demonstrates that swings between these two states take place on the same time-scales of luminosity variations of X-ray transients, and are therefore most easily interpreted in terms of changes in the rate of mass in-flow.

While accreting mass, the X-ray emission of IGR J18245-2452 varies dramatically on time-scales ranging from a second to a few hours. We interpret a state characterised by a lower flux and pulsed fraction, and by sudden increases of the hardness of the X-ray emission, in terms of the onset of a magnetospheric centrifugal inhibition of the accretion flow. Prospects of finding new members of the newly established class of transitional pulsars are also briefly discussed.
\end{abstract}

\section{Introduction}

The extremely short spin periods of millisecond pulsars are assumed to be the outcome of accretion of the mass transferred by a low mass $\left(\lesssim \mathrm{M}_{\odot}\right.$ ) companion star through an accretion disk [1]. This evolutionary framework is known as the recycling scenario of old neutron stars (NS) in binaries [2, 3]. After a Gyr-long mass accretion phase during which the system appears as a bright X-ray source, the mass transfer rate declines and allows the activation of a pulsar powered by the rotation of its magnetic field, emitting from the radio to the gamma-ray band. The $\sim 300$ millisecond radio pulsars (MSP) in our Galaxy are consequently believed to be the recycled descendants of accreting neutron stars in low mass X-ray binaries (NS-LMXB). The discovery of millisecond pulsations from accreting neutron stars in LMXB, so called accreting millisecond pulsars $[4,7,8]$, proved how mass accretion is able to effectively spin-up a neutron star to such fast rotational velocities.

Millisecond pulsars in binary systems are also ideal laboratories to study the interaction between the magnetosphere of a these quickly spinning neutron star, and the plasma transferred by the donor

a e-mail: papitto@ice.csic.es

This is an Open Access article distributed under the terms of the Creative Commons Attribution License 2.0, which permits unrestricted use, distribution, and reproduction in any medium, provided the original work is properly cited. 
companion star. Their observational appearance is in fact assumed to result from the balance between the outward pressure exerted by the pulsar radiation, and the inward ram pressure of the in-falling matter. Only when the pulsar emission manages to prevent the plasma to enter inside the light cylinder of the pulsar (with radius $R_{L C} \simeq 190 \mathrm{~km}$, for a NS spinning at $3.9 \mathrm{~ms}$ ) a rotation-powered pulsar is expected to turn on. On the contrary, when the in-falling matter squeezes the magnetosphere inside the light cylinder, and eventually reaches the neutron star surface, the system will appear as a bright X-ray source. It was then proposed that transient NS-LMXB would switch between rotation and accretion powered states as they alternate between X-ray outbursts and quiescence [5, 6, 9].

Here, we present the discovery of the first transitional system observed to swing between rotation and accretion powered pulsar states, definitely proving the evolutionary link shared by radio millisecond pulsars and NS-LMXB [31].

\section{IGR J18245-2452, a transitional accreting millisecond pulsar}

IGR J18245-2452 is a hard X-ray transient belonging to the globular cluster M28, first detected in outburst by ISGRI/INTEGRAL on 28 March 2013 [11]. At a distance of $5.5 \mathrm{kpc}$, the source attained an X-ray luminosity of $L \approx 3.5 \times 10^{36} \mathrm{erg} \mathrm{s}^{-1}$ in both soft $(0.5-10 \mathrm{keV})$, and hard (20-100 $\mathrm{keV}$ ) X-rays $[11,12]$. Observations of thermonuclear explosions caused by runaway burning of the material accreted onto the surface of a neutron star $[13,32]$ secured the identification of the compact object in the system. Coherent pulsations at a period of $3.9 \mathrm{~ms}$ were discovered in follow-up XMMNewton observations (see left panel of Fig. 1), making this source the fifteenth accreting millisecond pulsar discovered so far (the first by an observatory different than RXTE). The pulse profile could be modelled with two harmonic components, with average fractional amplitudes of 13.4(1) and 1.9(1) per cent, respectively (see inset of left panel of Fig. 1). Analysis of the Doppler shifts of the signal frequency revealed that the pulsar is in a $11.0 \mathrm{hr}$ circular orbit around $\mathrm{a} \approx 0.2 M_{\odot}$ companion star. The X-ray luminosity, the thermonuclear bursts, and the presence of a broad emission line at energies compatible with the K- $\alpha$ transition of iron, identify mass accretion as the driver of the pulsed emission observed from IGR J18245-2452.

Cross-referencing with catalogues of radio pulsars [23], it was realised that a source in M28, with the same spin and orbital parameters, had been already observed as a rotation powered radio pulsar a few years before, PSR J1824-2452I [10]. This proved that the source had performed a transition between the two states related by the recycling scenario of neutron stars, representing its most convincing observational evidence. The signal detected from this radio pulsar was faint (few tenths of $\mu \mathrm{Jy}$ at $2 \mathrm{GHz}$ ) and irregularly eclipsed by the presence of matter ejected by the pulsar wind.

The analysis of archival observations performed by Chandra revealed a past X-ray brightening by an order of magnitude, above a quiescent flux level of $10^{32} \mathrm{erg} \mathrm{s}^{-1}$ [31]. This is a strong indication that also at that time the matter transferred by the companion star started to spill out in the NS Roche lobe. However, the source was in a much dimmer state than during the outburst in 2013, and it is possible that accretion onto the neutron star surface was reduced or completely halted by magnetospheric centrifugal inhibition (i.e. a propeller state, [14]).

While the $2008 \mathrm{X}$-ray brightening lagged by two months the most recent previous detection of the radio pulsar, the reactivation of the radio pulsar after the 2013 X-ray outburst was even more prompt [31]. An observational campaign performed by the Green Bank, Parkes and Westerbork radio telescopes could detect a pulsed radio signal, already a few weeks after the latest detection of the Xray pulsar, and a couple of days since the last detection in X-rays (in which only an upper limit of 17 per cent of the pulse amplitude could be set). To illustrate the fast swing between the two states, we plot in the right panel of Fig. 1 a sample of the X-ray and radio observations of IGR J18245-2452/PSR J1824-2452I during the months of April and May 2013 (see [31] for the complete data-set). 

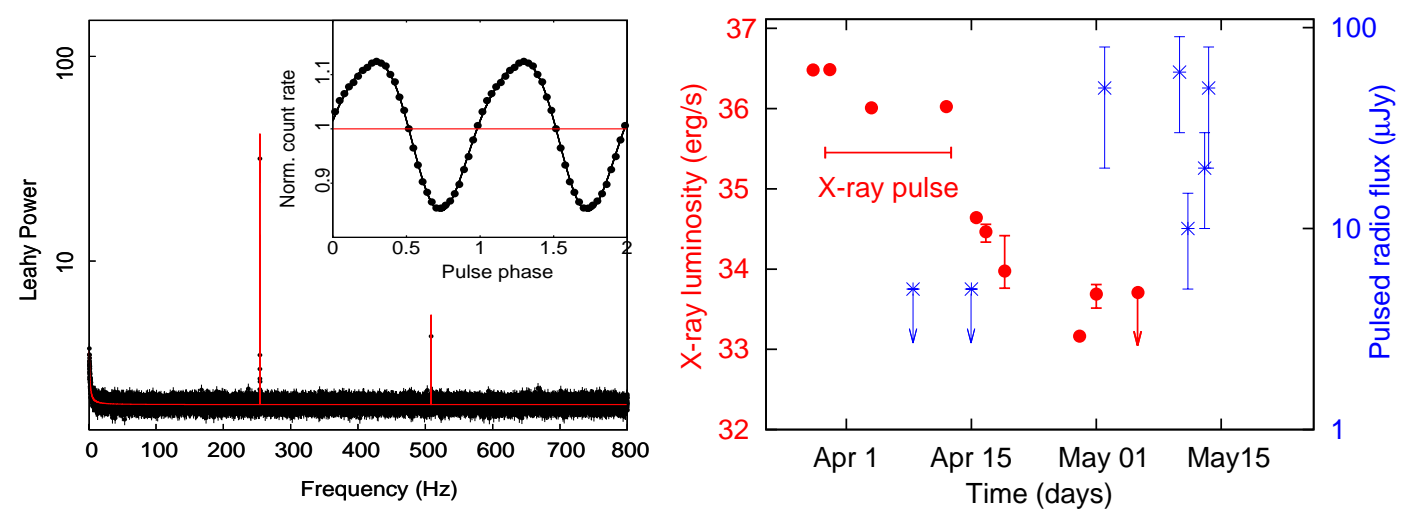

Figure 1. Left panel: Leahy normalised power spectrum obtained averaging $128 \mathrm{~s}$ intervals of XMM-Newton observations performed while IGR J18245-2452 was in outburst on 13 April 2013. The inset shows the pulse profile accumulated in the 0.5-10 keV energy band. Right panel: observed X-ray luminosity (left scale, red dots) and pulsed radio flux (right scale, blue asterisks) of IGR J18245-2452/PSR J1824-2452I during observations performed between 28 March and 15 May 2013. The X-ray luminosity points refer to observations performed by INTEGRAL/ISGRI, Swift/XRT, XMM-Newton/EPICpn and Chandra, and are evaluated in the $0.5-10 \mathrm{keV}$ band for a distance of $5.5 \mathrm{kpc}$. Radio pulsed flux were evaluated at $2 \mathrm{GHz}$ (Green Bank Radio Telescope) and $1.4 \mathrm{GHz}$ (WSRT/Parkes). Only a subset of the available observations is shown, for illustrative purposes. See [31] for the complete data set.

The swings observed from IGR J18245-2452 proved that transitions between accretion and rotation powered states may take place on timescales comparable to those characterising the alternation of X-ray outbursts and quiescence in transient LMXB. When the system is found in X-ray quiescence $\left(L_{X} \approx 10^{32} \mathrm{erg} \mathrm{s}^{-1}\right.$ ), the rate of mass accretion onto the neutron star is lower than $10^{-14} \mathrm{M}_{\odot} \mathrm{yr}^{-1}$. The pressure exerted by the emission of a $3.9 \mathrm{~ms}$ rotation-powered pulsar with a field of $10^{8}-10^{9} \mathrm{G}$ (typical of MSP) is able to keep the plasma beyond the light cylinder, and to account for the observed $\mathrm{X}$-ray luminosity. An increase of the mass inflow rate may then push the magnetosphere inside the light cylinder, switching off the radio pulsed emission. A bright $\left(L_{X} \approx 10^{36} \mathrm{erg} \mathrm{s}^{-1}\right) \mathrm{X}$-ray outburst is subsequently triggered after enough mass has been loaded in the disk [15], and the magnetosphere is squeezed to a size of the order of the co-rotation radius ( $\approx 40 \mathrm{~km}$ in IGR J18245-2452). Our observations showed that as the mass accretion rate decreased at the end of the outburst, a rotation-powered radio pulsar reactivated at most two weeks after the last detection as an X-ray pulsar, and a couple of days after the last detection in X-rays at a luminosity (few $\times 10^{33} \mathrm{erg} \mathrm{s}^{-1}$ ) above the quiescent level.

\subsection{The X-ray variability}

During the X-ray outburst shown in 2013, IGR J18245-2452 shows a marked and peculiar variability of the magnitude and energy spectral distribution of its X-ray emission (Ferrigno et al. 2013, A\&A, submitted; see the inset of left panel of Fig. 2 for the light curve observed by XMM-Newton). A similar variability characterises also the radio flux observed at 5.5 and $9 \mathrm{GHz}$ by ATCA [16]. The power spectrum of the X-ray emission is dominated by a low frequency flicker noise component, described by a power law, $P(f) \propto f^{-1.2}$, cut-off at $\approx$ few $\times 10^{-4} \mathrm{~Hz}$ (see left panel of Fig. 2). Such a time-scale is compatible with the dynamical time scale at the outer radius of a viscous disk in IGR $\mathrm{J} 18245-2452$, and possibly indicates the propagation of fluctuations originating in the whole disk 

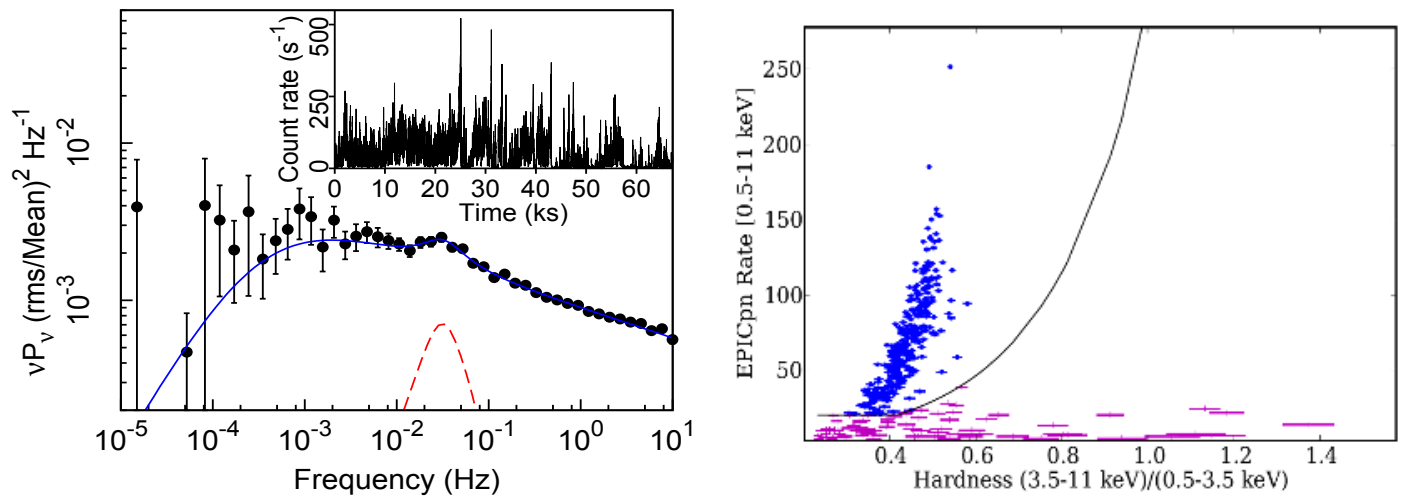

Figure 2. Left panel: power density spectrum of a 67 ks XMM-Newton observation of IGR J18245-2452 performed on 13 April 2013, re-binned geometrically using a factor of 1.3, and subtracted of the white noise level. The inset shows the 0.5-10 keV light curve. Right panel: hardness-intensity diagram of IGR J18245-2452.

down to the inner disk [17], as a possible explanation for the observed variability. This is at odds with the properties of low frequency variability usually observed from accreting millisecond pulsars, which show a noise with a cut-off at a frequency in the $0.1-1 \mathrm{~Hz}$ range [18].

The source follows two branches in a hardness-intensity diagram (see right panel of Fig. 2). In the one marked by blue points, the hardness varies in a relatively tight interval (0.3-0.5), in correlation with the variations of the X-ray count rate between 30 and 250 counts per second. In the branch marked by magenta points, the hardness performs swings up to $\approx 1.4$ at a roughly constant, and lower count rate level $\left(\sim 20\right.$ counts $\left.\mathrm{s}^{-1}\right)$. Considering that the pulsed fraction is tightly correlated with the $\mathrm{X}$-ray flux, thus lower when the source is in the magenta branch, this low state can be tentatively interpreted in terms of the onset of a centrifugal barrier (i.e., a propeller state [14]). According to this qualitative interpretation, the decrease of the X-ray flux and of the pulsed fraction would be due to the (partial) inhibition of accretion onto the neutron star surface, and in particular of the fraction of matter falling close to the magnetic poles. The hardening of the spectrum would be related to the less efficient cooling of a Comptonizing cloud due to the lower flux of seed photons coming from the NS surface, and/or to the energy deposited in the inner regions of the disk by a propellering magnetosphere [19].

\section{Future prospects}

IGR J18245-2452 is the first member of a new class of pulsars, swinging between rotation and accretion powered pulsar states, on timescales short enough to make the transitions actually observable. More members of this class should be searched among those binaries in which the companion star currently fills its Roche lobe and spills out matter towards the neutron star. The rate of mass transfer from the donor star varies in fact over much longer timescales ( $\gtrsim 50 \mathrm{Myr}$; e.g., [20]).

The irregular eclipses of the pulsed emission observed from a number of millisecond radio pulsars, as well as the variations of their dispersion measure, are clear indications of pulsars whose radiation is ejecting the matter that the companion tries to transfer. These radio pulsars are usually dubbed as black-widow $\left(M_{c}<<0.1 \mathrm{M}_{\odot}\right.$; [21]) or redbacks $\left(M_{c} \sim 0.2-0.4 \mathrm{M}_{\odot}\right.$; [22]), depending on the mass of the companion star. Indeed, IGR J18245-2452 is a redback, as well as the other known 


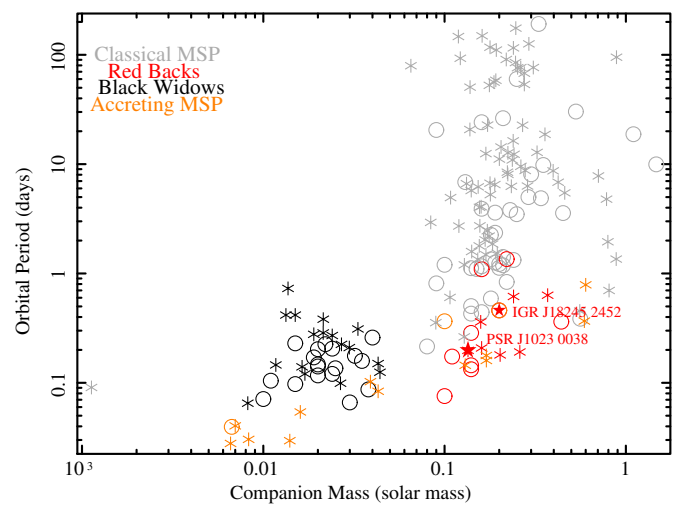

Figure 3. Orbital period and minimum companion mass of field classical MSP with a white dwarf companion (grey), black widows (black), redbacks (red), and accreting millisecond pulsars (orange). Asterisks and circles mark sources in the Galactic field and in globular clusters, respectively.

transitional system, PSR J1023+0038, a radio pulsar which showed optical emission lines in 20002001 , indicating the presence of an accretion disk at that time [24] ${ }^{1}$. This strongly indicates that transitions to the accretion phase are expected to happen among pulsars that are vaporising their companion. The number of systems of this class is rapidly increasing during the last few years [25], as they are bright gamma-ray emitters and are easily detected by Fermi/LAT, increasing the probability of finding new transitional systems in the near future.

On the other hand, during X-ray quiescence accreting millisecond pulsars are mostly indistinguishable from rotation-powered pulsars, at least for what concerns the irradiation of the companion star, and their spin and orbital evolution [26-30]. In addition, accreting millisecond pulsars populate the same region of the plot of orbital periods and companion star mass than black-widow and redback (see Fig. 3). However, searches for a radio [33, 34] and gamma-ray [35] have not been successful, so far. These non-detections were possibly due to observational biases (e.g. the larger impact of absorption of a radio signal by matter enshrouding the system, in close binary systems) or to a paucity of gamma-ray photons. Future observations with a longer exposure will be therefore crucial to firmly assess the behaviour of these sources during quiescence.

\section{Acknowledgements}

Work done in the framework of the grants AYA2012-39303, as well as SGR2009-811, and iLINK2011-0303. AP is supported by a Juan de la Cierva Research Fellowship.

\section{References}

[1] Bisnovatyi-Kogan, G. S., \& Komberg, B. V. 1974, SvA, 18, 217

[2] M.A. Alpar, A.F. Cheng, M.A. Ruderman, J. Shaham, Nature, 300, 728 (1982)

[3] V. Radhakrishnan, G. Srinivasan, Current Science 51, 1096 (1982)

[4] D. Chakrabarty, E.H. Morgan, Nature, 394, 346 (1998)

\footnotetext{
${ }^{1}$ The system had an X-ray luminosity $\lesssim 10^{34} \mathrm{erg} \mathrm{s}^{-1}$ at that time, so that accretion onto the neutron star surface was not taking place at a high rate, and the system was presumably in a propeller state.
} 
[5] L. Stella, S. Campana, M. Colpi, S. Mereghetti, M. Tavani, ApJL, 423, L47 (1994)

[6] S. Campana, M. Colpi, S. Mereghetti, L. Stella, M. Tavani, A\&A, 8, 279 (1998)

[7] Wijnands, R., \& van der Klis, M. 1998, Nature, , 394, 344

[8] Patruno, A., \& Watts, A. L. 2012, arXiv:1206.2727

[9] Burderi, L., Possenti, A., D’Antona, F., et al. 2001, ApJL, , 560, L71

[10] S. Begin, Master of science, The Faculty of Graduate Studies (Physics), The University of British Columbia (2006)

[11] D. Eckert, M. Del Santo, A. Bazzano, K. Watanabe, A. Paizis, E. Bozzo, C. Ferrigno, I. Caballero, L. Sidoli, L. Kuiper, The Astronomer's Telegram 4925, 1 (2013)

[12] P. Romano, S.D. Barthelmy, D.N. Burrows, V. D'Elia, N. Gehrels, S.T. Holland, J.A. Kennea, C.B. Markwardt, F.E. Marshall, K.L. Page, The Astronomer's Telegram 4929, 1 (2013)

[13] M. Linares, The Astronomer's Telegram 4960, 1 (2013)

[14] A.F. Illarionov, R.A. Sunyaev, A\&A, 39, 185 (1975)

[15] J. van Paradijs, ApJL, 464, L139 (1996)

[16] L. Pavan, G. Wong, M.H. Wieringa, N.F.H. Tothill, M.D. Filipovic, E. Bozzo, C. Ferrigno, A. Papitto, P. Romano, The Astronomer's Telegram 4981, 1 (2013)

[17] Y.E. Lyubarskii, MNRAS, 292, 679 (1997)

[18] S. van Straaten, M. van der Klis, R. Wijnands, ApJ, 619, 455 (2005), astro-ph/0410505

[19] S.N. Zhang, W. Yu, W. Zhang, ApJL, 494, L71 (1998)

[20] T.M. Tauris, N. Langer, M. Kramer, MNRAS, 425, 1601 (2012), 1206.1862

[21] A.S. Fruchter, D.R. Stinebring, J.H. Taylor, Nature, 333, 237 (1988)

[22] N. D’Amico, A. Possenti, R.N. Manchester, J. Sarkissian, A.G. Lyne, F. Camilo, ApJL, 561, L89 (2001)

[23] Manchester, R. N., Hobbs, G. B., Teoh, A., \& Hobbs, M. 2005, VizieR Online Data Catalog, 7245, 0

[24] A.M. Archibald, I.H. Stairs, S.M. Ransom, V.M. Kaspi, V.I. Kondratiev, D.R. Lorimer, M.A. McLaughlin, J. Boyles, J.W.T. Hessels, R. Lynch et al., Science 324, 1411 (2009)

[25] M.S.E. Roberts, Surrounded by spiders! New black widows and redbacks in the Galactic field, in IAU Symposium (2013), Vol. 291 of IAU Symposium, pp. 127-132

[26] L. Burderi, T. Di Salvo, F. D’Antona, N.R. Robba, V. Testa, A\&A, 404, L43 (2003)

[27] T. di Salvo, L. Burderi, A. Riggio, A. Papitto, M.T. Menna, MNRAS, 389, 1851 (2008)

[28] J.M. Hartman, A. Patruno, D. Chakrabarty, D.L. Kaplan, C.B. Markwardt, E.H. Morgan, P.S. Ray, M. van der Klis, R. Wijnands, ApJ, 675, 1468 (2008)

[29] A. Patruno, ApJ, 722, 909 (2010)

[30] A. Papitto, A. Riggio, L. Burderi, T. di Salvo, A. D’Aí, R. Iaria, A\&A, 528, A55 (2011)

[31] Papitto, A., Ferrigno, C., Bozzo, E., et al. 2013, Nature, , 501, 517

[32] Papitto, A., Bozzo, E., Ferrigno, C., et al. 2013, The Astronomer's Telegram, 4959, 1

[33] M.N. Iacolina, M. Burgay, L. Burderi, A. Possenti, T. di Salvo, A\&A, 497, 445 (2009)

[34] M.N. Iacolina, M. Burgay, L. Burderi, A. Possenti, T. di Salvo, A\&A, 519, A13 (2010)

[35] Y. Xing, Z. Wang, ApJ, 769, 119 (2013) 